\title{
Macrovascular Complications and Prevalence of Urgency Incontinence in Japanese Patients with Type 2 Diabetes Mellitus: The Dogo Study
}

\author{
Shinya Furukawa ${ }^{1,2}$, Takenori Sakai ${ }^{3}$, Tetsuji Niiya ${ }^{4}$, Hiroaki Miyaoka $^{5}$, Teruki Miyake ${ }^{6}$, \\ Shin Yamamoto ${ }^{6}$, Sayaka Kanzaki ${ }^{6}$, Koutatsu Maruyama ${ }^{7}$, Keiko Tanaka ${ }^{1,2}$, Teruhisa Ueda ${ }^{8}$, \\ Hidenori Senba ${ }^{1,6}$, Masamoto Torisu ${ }^{9}$, Hisaka Minami ${ }^{10}$, Morikazu Onji ${ }^{11}$, Takeshi Tanigawa ${ }^{7}$, \\ Bunzo Matsuura $^{12}$, Yoichi Hiasa ${ }^{6}$ and Yoshihiro Miyake ${ }^{1,2}$
}

\begin{abstract}
Objective Macrovascular diseases and urgency incontinence are common among Japanese patients with type 2 diabetes mellitus. However, little evidence exists regarding the association between stroke and urgency incontinence among patients with type 2 diabetes mellitus. We examined the associations between macrovascular complications and urgency incontinence among Japanese patients with type 2 diabetes mellitus.

Methods The study subjects were 818 Japanese patients with type 2 diabetes mellitus. Urgency incontinence was defined as present when a subject answered "once a week or more" to the question: "Within one week, how often do you leak urine because you cannot defer the sudden desire to urinate?" We adjusted our analyses for sex, age, body mass index, duration of type 2 diabetes, current smoking, current drinking, hypertension, dyslipidemia, glycated hemoglobin, diabetic nephropathy, diabetic retinopathy, and diabetic peripheral neuropathy.

Results The prevalence of urgency incontinence was 9.2\%. Stroke was independently positively associated with urgency incontinence, with an adjusted odds ratio of 2.34 (95\% confidence interval: 1.03-4.95). The associations between ischemic heart disease or peripheral artery disease and the prevalence of urgency incontinence were not significant.

Conclusion In Japanese patients with type 2 diabetes mellitus, stroke, but not ischemic heart diseases or peripheral artery disease, was independently positively associated with urgency incontinence.
\end{abstract}

Key words: diabetes, incontinence, Japanese, stroke, macrovascular complications

(Intern Med 56: 889-893, 2017)

(DOI: 10.2169/internalmedicine.56.8063)

\section{Introduction}

Subjects with urgency incontinence have worse scores on quality-of-life and depression scales, poorer quality of sleep, and lower work productivity than do matched controls $(1,2)$. The prevalence values of urgency incontinence among patients with type 2 diabetes mellitus were 1.9-

\footnotetext{
${ }^{1}$ Department of Epidemiology and Preventive Medicine, Ehime University Graduate School of Medicine, Japan, ${ }^{2}$ Epidemiology and Medical Statistics Unit, Translational Research Center, Ehime University Hospital, Japan, ${ }^{3}$ Department of Internal Medicine, Yawatahama General City Hospital, Japan, ${ }^{4}$ Department of Internal Medicine, Matsuyama Shimin Hospital, Japan, ${ }^{5}$ Department of Internal Medicine, Saiseikai Matsuyama Hospital, Japan, ${ }^{6}$ Department of Gastroenterology and Metabology, Ehime University Graduate School of Medicine, Japan, ${ }^{7}$ Department of Public Health, Juntendo University School of Medicine, Japan, ${ }^{8}$ Department of Internal Medicine, Ehime Prefectural Central Hospital, Japan, ${ }^{9}$ Department of Internal Medicine, Saiseikai Saijo Hospital, Japan, ${ }^{10}$ Department of Internal Medicine, Ehime Niihama Hospital, Japan, ${ }^{11}$ Department of Internal Medicine, Saiseikai Imabari Hospital, Japan and ${ }^{12}$ Department of Lifestyle-related Medicine and Endocrinology, Ehime University Graduate School of Medicine, Japan
}

Received for publication July 28, 2016; Accepted for publication August 15, 2016

Correspondence to Dr. Shinya Furukawa, shinya.furukawa@gmail.com 
$26.4 \%$ in previous studies (3-11). In a Korean case-control study, urgency incontinence scores among cases with type 2 diabetes mellitus were higher than those among a control group (12). In an Italian case-control study, the prevalence of urgency incontinence among cases with type 2 diabetes mellitus were significantly higher than those of a healthy control (6). Diabetes was found to be significantly positively associated with urgency incontinence in a US study of 121,701 women (3), in a Norwegian study of 21,057 women (9), and in a US study of 2,763 postmenopausal women (12).

The prevalence of urgency incontinence among patients with stroke was $15.7-32.5 \%(13-15)$. Stroke was significantly positively associated with urgency incontinence in a US study of 3,809 elderly subjects (16). Prospective studies have demonstrated that diabetes mellitus was a risk factor for stroke $(17,18)$. However, little evidence exists regarding the association between stroke and urgency incontinence among patients with type 2 diabetes mellitus. In a US study of 2,994 women with type 2 diabetes mellitus, the prevalence of stroke among diabetic patients with weekly urinary incontinence was similar to that of patients with less than weekly urinary incontinence or no incontinence (19). In a Taiwanese study of 279 patients with type 2 diabetes mellitus, the association between stroke history and urgency incontinence among patients with type 2 diabetes mellitus was not significant (8).

In the present study, we examined the association between macrovascular complications and urgency incontinence among Japanese patients with type 2 diabetes mellitus.

\section{Materials and Methods}

\section{Study population}

The Dogo Study is a multicenter prospective cohort study that recruited 1,051 Japanese patients with previously diagnosed type 2 diabetes mellitus from September 2009 to September 2014 (median age at recruitment, 61.6 years; range, 19-88 years; $60.9 \%$ men). Collaborating physicians from 10 hospitals, who specialized in diabetes mellitus, were responsible for the diagnosis of type 2 diabetes mellitus as defined by the Japan Diabetes Society criteria. Excluded from our current analysis were 233 patients who had missing data on the variables under study. Thus, the final analysis sample consisted of 818 patients. The present study protocol received ethical approval from the institutional review board of the Ehime University Graduate School of Medicine. Written informed consent was obtained from all patients enrolled in the study.

\section{Measurements}

Each participant completed a self-administered questionnaire, which collected data on diabetes duration, current smoking habits, current drinking habits, use of antihypertensive medication, use of anti-hyperlipidemic medication, height, and weight. Each patient's body mass index (BMI) was calculated as their weight $(\mathrm{kg})$ divided by the square of their height $\left(\mathrm{m}^{2}\right)$. Current smoking was defined as positive if a study subject reported smoking at least one cigarette per day. Current drinking was defined as present when subjects reported drinking habitually. Blood pressure was measured with a cuff in the sitting position, after a rest period of greater than 5 minutes. Hypertension was defined as positive if systolic blood pressure was $>140 \mathrm{mmHg}$, diastolic blood pressure was $>90 \mathrm{mmHg}$, or both, or if the patient had received anti-hypertensive medication. Dyslipidemia was defined as positive if the serum total cholesterol concentration was $>220 \mathrm{mg} / \mathrm{dL}(5.69 \mathrm{mmol} / \mathrm{L})$, triglyceride concentration was $>150 \mathrm{mg} / \mathrm{dL}(1.69 \mathrm{mmol} / \mathrm{L})$, high-density lipoprotein cholesterol concentration was $<40 \mathrm{mg} / \mathrm{dL}(1.03 \mathrm{mmol} / \mathrm{L})$, or if the patients were already being treated with lipid-lowering agents.

\section{Assessing macrovascular complications}

Stroke and ischemic heart disease were defined based on the self-administered questionnaires, medical records, and/or admission data. Peripheral artery diseases were defined based on self-administered questionnaires, ankle brachial in$\operatorname{dex}(\mathrm{ABI}) \leq 0.9$, medical records, and/or admission data. ABI values were measured using the Vasera VS-100 (Fukuda Denshi Co., LTD., Tokyo, Japan) and/or BP-203 RPEIII (Omron Co., LTD., Kyoto, Japan).

\section{Assessing type 2 diabetes mellitus complications}

Microvascular complications of type 2 diabetes mellitus included retinopathy, nephropathy, and neuropathy. Retinopathy was diagnosed based on the presence of hemorrhaging, microaneurysm, soft and hard exudates, areas of neovascularization, or laser coagulation scars in at least one eye. A diagnosis was conducted using florescence fundoscopy on dilated pupils within three months of recruitment. Several ophthalmology specialists were responsible for evaluating the fundus of all participants. The estimated glomerular filtration rate (eGFR) was calculated using serum creatinine $(\mathrm{Cr})$ : $194 \times$ serum $\mathrm{Cr}^{-1.094} \times$ age $\mathrm{a}^{-0.287}$ (if female $\times$ 0.739) (20). Diabetic nephropathy was defined as positive when the urine albumin-to-creatinine ratio was $\geq 30 \mathrm{mg} / \mathrm{g}$ creatinine and/or eGFR was $<30 \mathrm{~mL} / \mathrm{min} / 1.73 \mathrm{~m}^{2}$ (21). Diabetic peripheral neuropathy was diagnosed if the patients showed two or more of the following three characteristics: neuropathic symptoms (numbness, pain, paresthesia, or decreased sensation in the tips of the toes and bottoms of the feet), an absence of the Achilles reflex, or abnormal vibration perception threshold scores assessed with a $128 \mathrm{-Hz}$ tuning fork (22).

\section{Assessing urgency incontinence}

Study subjects were considered to have urgency incontinence if they answered "once a week or more" to the question: "Within one week, how often do you leak urine because you cannot defer the sudden desire to urinate?" 
Table 1. Characteristics of the 818 Study Participants.

\begin{tabular}{lc}
\hline Variable & $\mathrm{n}(\%)$ \\
\hline Age, years, mean \pm SD & $61.9 \pm 11.1$ \\
Male gender (\%) & $514(62.8)$ \\
BMI, kg/m ${ }^{2}$, mean \pm SD & $25.2 \pm 4.8$ \\
HbAlc, \%, mean \pm SD & $7.89 \pm 1.85$ \\
Insulin therapy (\%) & $217(26.5)$ \\
Duration of T2DM, years, mean \pm SD & $11.1 \pm 10.2$ \\
Current drinking (\%) & $333(40.7)$ \\
Current smoking (\%) & $148(18.1)$ \\
Hypertension (\%) & $574(70.2)$ \\
Hyperlipidemia (\%) & $618(75.6)$ \\
& \\
Microvascular complications & \\
Diabetic peripheral neuropathy (\%) & $505(61.7)$ \\
Diabetic retinopathy (\%) & $239(29.2)$ \\
Diabetic nephropathy (\%) & $270(33.0)$ \\
& \\
Macrovascular complications & \\
Stroke (\%) & $56(6.9)$ \\
Ischemic heart disease (\%) & $89(10.9)$ \\
Peripheral arterial disease (\%) & $59(7.2)$ \\
Urgency incontinence (\%) & $75(9.2)$ \\
\hline BMI: body mass index, T2DM: type 2 diabetes mellitus
\end{tabular}

\section{Statistical analysis}

Estimations of crude odds ratios (ORs) and their 95\% confidence intervals (CIs) were performed using logistical regression analyses for urgency incontinence in relation to macrovascular complications. Sex, age, body mass index, duration of type 2 diabetes, current smoking, current drinking, hypertension, dyslipidemia, glycated hemoglobin, diabetic nephropathy, diabetic retinopathy, and diabetic peripheral neuropathy were selected as potential confounding factors. Multiple regression logistic analyses were used to adjust for potential confounding factors. All statistical analyses were performed using the SAS software package version 9.4 (SAS Institute Inc., Cary, NC, USA).

\section{Results}

Among the 818 patients with type 2 diabetes mellitus, the mean age of the study participants was 61.9 years, and the prevalence of urgency incontinence was $9.2 \%$. The prevalence values of stroke, ischemic heart disease, and peripheral artery disease were $6.9 \%, 10.9 \%$, and $7.2 \%$, respectively (Table 1). Table 2 shows crude and adjusted ORs and 95\% CIs for the prevalence of urgency incontinence in relation to macrovascular complications. The prevalence of urgency incontinence among diabetic patients with stroke was $17.9 \%$, while the prevalence of urgency incontinence among those without stroke was $8.5 \%$. In the crude analysis, stroke and peripheral arterial disease were positively associated with urgency incontinence. After adjustment for sex, age, BMI, duration of type 2 diabetes, current smoking, current drinking, hypertension, dyslipidemia, glycated hemoglobin, diabetic nephropathy, diabetic retinopathy, and diabetic peripheral neuropathy, stroke was independently positively associated with urgency incontinence: the adjusted OR was 2.34 (95\% CI: 1.03-4.95). The association between peripheral arterial disease and urgency incontinence disappeared. No evident relationships were found between ischemic heart disease or peripheral artery disease and urgency incontinence.

\section{Discussion}

To our knowledge, this is the first study to show that stroke was found to be independently positively associated with urgency incontinence among Japanese patients with type 2 diabetes mellitus.

In an Italian study of 80 patients with ischemic cerebrovascular disease, the prevalence of urgency incontinence was $32.5 \%$ (13). In a Japanese study of 500 patients with chronic-phase stroke, the prevalence of urgency incontinence was $28.2 \%$ (14). In a Norwegian study of 315 patients with acute stroke, the cumulative incidence of urgency incontinence was $13.9 \%$ within three months from the onset of stroke (15). In the present study, the prevalence of urgency incontinence among diabetic subjects with stroke was $15.7 \%$.

Among patients with type 2 diabetes mellitus, the association between macrovascular complications and urgency incontinence is still unclear. In a Taiwanese study of 279 patients with type 2 diabetes mellitus, the association between stroke history and urgency incontinence was not significant (OR 2.55, p=0.082) (8). In a US study of 2,994 women with type 2 diabetes mellitus, the prevalence of stroke among diabetic patients with weekly urinary incontinence was similar to that of patients with less than weekly urinary incontinence or no incontinence (19). These findings are inconsistent with the present results. The discrepancies among studies may be explained, at least in part, by differences in the sample size; characteristics such as age, sex, race, BMI, and prevalence of stroke; and the confounding factors considered. Stroke may directly injure neuro-micturition pathways (23). Dysfunction of the prefrontal cortex or limbic system may cause urgency incontinence: the limbic system prevents urgency incontinence via suppression of unwanted emotional reactions, and activation of the prefrontal cortex is important for maintaining voluntary control of voiding (24). Several pieces of evidence exist regarding the association between imaging findings in the brain and urgency incontinence. In a US study of 99 elderly adults, the volume of white matter change in the brain was positively associated with urgency incontinence (25). In the previously cited Japanese study of 500 patients with chronic-phase stroke, the grading of deep white matter hyperintensity was positively associated with urgency incontinence (14). In a US study of 97 elderly subjects, the presence of white matter change in the right frontal and right inferior frontal regions predicted incontinence severity (26). In a Japanese study of 63 elderly subjects, the association between white matter lesions on magnetic resonance imaging and severe urgency inconti- 
Table 2. Crude and Adjusted Odds Ratios and 95\% Confidence Intervals for Urgency Incontinence in Relation to Macrovascular Complications.

\begin{tabular}{|c|c|c|c|}
\hline Variable & Prevalence $(\%)$ & Crude OR $(95 \% \mathrm{CI})$ & Adjusted OR $(95 \% \mathrm{CI})$ \\
\hline \multicolumn{4}{|l|}{ Stroke } \\
\hline No & $65 / 762(8.5)$ & 1.00 & 1.00 \\
\hline Yes & $10 / 56(17.9)$ & $2.33(1.07-4.66)$ & $2.34(1.03-4.95)$ \\
\hline \multicolumn{4}{|c|}{ Ischemic heart disease } \\
\hline No & $68 / 729(9.3)$ & 1.00 & 1.00 \\
\hline Yes & $7 / 89(7.9)$ & $0.83(0.34-1.75)$ & $0.65(0.26-1.42)$ \\
\hline \multicolumn{4}{|c|}{ Peripheral arterial disease } \\
\hline No & $65 / 759(8.6)$ & 1.00 & 1.00 \\
\hline Yes & $10 / 59(17.0)$ & $2.18(1.001-4.34)$ & $1.78(0.78-3.74)$ \\
\hline
\end{tabular}

nence was significant (27). The association between ischemic heart disease and urgency incontinence is still unclear.

Our results are consistent with those of a Taiwanese study of 279 patients with type 2 diabetes mellitus (8) and of a US study of 2,994 women with type 2 diabetes mellitus showing no association between ischemic heart disease and urgency incontinence (19). To our knowledge, no evidence exists regarding the association between peripheral artery disease and urgency incontinence.

Several limitations associated with the present study warrant mention. First, this was a cross-sectional study; therefore, we cannot conclude that there is a causal relationship between stroke and urgency incontinence. Second, we were unable to diagnose benign prostate hyperplasia because we were unable to measure the size of the prostate and postvoid residual volume. Third, data on brain imaging in the present study were not available. Fourth, data on history of hysterectomy or prostatectomy were not available in the present study. Fifth, the definition of stroke was based on the self-administered questionnaires, medical records, and/or admission data. We could not separate the type of stroke. Similarly, the definition of ischemic heart diseases was based on the self-administered questionnaires, medical records, and/or admission. We might have underestimated the prevalence of ischemic heart disease. As the prevalence values of asymptomatic myocardial ischemia were $34-42 \%$ among patients with diabetes (28). The misclassification regarding stroke and ischemic heart disease might affect the association between macrovascular complications and urgency incontinence. Sixth, we did not assess the autonomic nervous function. As diabetic autonomic neuropathy may cause urgency incontinence. Finally, all data on urgency incontinence were self-reported. However, previous studies have established the reliability and validity of self-reported urinary incontinence (3).

In conclusion, stroke, but not ischemic heart diseases or peripheral artery disease, was found to be significantly positively associated with urgency incontinence in Japanese patients with type 2 diabetes mellitus.
The authors state that they have no Conflict of Interest (COI).

\section{Acknowledgement}

We thank Eriko Kawamoto from University of the Ryukyu, Keiko Kikuchi and Tomo Kogama from Ehime University, and Eri Furukawa from the Furukawa Clinic for their valuable help and advice.

\section{Funding}

This study was supported by JSPS KAKENHI (Grant Numbers 21790583 and 23790697).

\section{References}

1. Stewart WF, Van Rooyen JB, Cundiff GW, et al. Prevalence and burden of overactive bladder in the United States. World J Urol 20: 327-336, 2003.

2. Hu TW, Wagner TH, Bentkover JD, et al. Estimated economic costs of overactive bladder in the United States. Urology 61: 1123-1128, 2003.

3. Danforth KN, Townsend MK, Curhan GC, et al. Type 2 diabetes mellitus and risk of stress, urge and mixed urinary incontinence. $\mathrm{J}$ Urol 181: 193-197, 2009.

4. Izci Y, Topsever P, Filiz TM, et al. The association between diabetes mellitus and urinary incontinence in adult women. Int Urogynecol J 20: 947-952, 2009.

5. Ho CH, Tai HC, Yu HJ. Urodynamic finding in female diabetic patients with and without overactive bladder symptoms. Neurourol Urodyn 29: 424-427, 2010.

6. Palleschi G, Pastore AL, Maggioni C, et al. Overactive bladder in diabetes mellitus patients: a questionnaire-based observation investigation. World J Urol 32: 1021-1025, 2014.

7. Liu RT, Chung MS, Lee WC, et al. Prevalence of overactive bladder and associated risk factors in 1,359 patients with type 2 diabetes. Urology 78: 1040-1045, 2011.

8. Chiu AF, Huang MH, Wang CC, et al. Higher glycoslated hemoglobin levels increase the risk of oberactive bladder syndrome in patients with type 2 diabetes mellitus. Int J Urol 19: 995-1001, 2012.

9. Ebbesen MH, Hannestad YS, Midthjell K, et al. Diabetes and urinary incontinence - prevalence data from Norway. Acta Obstet Gynecol Scand 86: 1256-1262, 2007.

10. Tai HC, Chung SD, Ho CH, et al. Metabolic syndrome components worsen lower urinary tract symptoms in women with type 2 diabetes. J Clin Endocrinol Metab 95: 1143-1150, 2010.

11. Brown JS, Vittinghoff E, Lin F, et al. Prevalence and risk factors 
for urinary incontinence in women with type 2 diabetes and impaired fasting glucose: findings from the National Health and $\mathrm{Nu}-$ trition Examination Survey (NHANES) 2001-2002. Diabetes Care 29: 1307-1312, 2006.

12. Bang WJ, Lee JY, Koo KC, et al. Is type-2 diabetes mellitus associated with overactive bladder symptoms in men with lower urinary tract symptoms? Urology 84: 670-674, 2014.

13. Arena MG, Di Rosa AE, Arcudi L, et al. Voiding disorders in patients with cerebrovascular disease. Funct Neurol 7: 47-49, 1992.

14. Itou Y, Yamada S, Konoeda F, et al. Burden of overactive bladder symptom on quality of life in stroke patients. Neurourol Urodyn 32: 428-434, 2013.

15. Pettersen R, Wyller TB. Prognostic significance of micturition disturbance after acute stroke. J Am Geriatr Soc 54: 1878-1884, 2006.

16. Wetle T, Scherr P, Branch LG, et al. Difficulty with holding urine among older persons in a geographically defined community: prevalence and correlates. J Am Geriatr Soc 43: 349-355, 1995.

17. Cui R, Iso H, Yamagishi K, et al. Diabetes mellitus and risk of stroke and its subtypes among Japanese. Stroke 42: 2611-2614, 2011.

18. Emerging Risk Factors Collaboration, Sarwar N, Gao P, Seshasai $\mathrm{SR}$, et al. Diabetes mellitus, fasting blood glucose concentration, and risk of vascular disease: a collaborative meta-analysis of 102 prospective studies. Lancet 375: 2215-2222, 2010.

19. Phelan S, Kanaya AM, Subak LL, et al. Prevalence and risk factors for urinary incontinence in overweight and obese diabetic women: action for health in diabetes (look ahead) study. Diabetes Care 32: 1391-1397, 2009.

20. Matsuo S, Imai E, Horio MY, et al. Collaborators developing the Japanese equation for estimated GFR. Revised equations for estimated GFR from serum creatinine in Japan. Am J Kidney Dis $\mathbf{5 3}$ : 982-992, 2009.

21. Haneda M, Utsunomiya K, Koya D, et al. A new classification of diabetic nephropathy 2014: a report from Joint Committee on diabetic nephropathy. J Diabetes Investig 6: 242-246, 2015.
22. Yasuda H, Sanada M, Kitada K, et al. Rationale and usefulness of newly devised abbreviated diagnostic criteria and staging for diabetic polyneuropathy. Diabetes Res Clin Pract 77: S178-S183, 2007.

23. Gelber DA, Good DC, Laven LJ, et al. Causes of urinary incontinence after acute hemispheric stroke. Stroke 24: 378-382, 1993.

24. Griffiths D, Tadic SD. Bladder control, urgency, and urge incontinence: evidence from functional brain imaging. Neurourol Urodyn 27: 466-474, 2008.

25. Wakefield DB, Moscufo N, Guttmann CR, et al. White matter hyperintesoties predict functional decline in voiding, morbility, and cognition in older adults. J Am Geriatr Soc 58: 275-281, 2010.

26. Kuchel GA, Moscufo N, Guttmann CR, et al. Localization of brain white matter hyperintensities and urinary incontinence in community-dwelling older adults. J Gerontol A Biol Sci Med Sci 64: 902-909, 2009.

27. Sakakibara R, Hattori $T$, Uchiyama $T$, et al. Urinary function in elderly people with and without leukoaraiosis: relation to cognitive and gait function. J Neurol Neurosurg Psychiatry 67: 658-660, 1999.

28. Levine GN, Bates ER, Blankenship JC, et al. American College of Cardiology Foundation, American Heart Association Task Force on Practice Guidelines, Society for Cardiovascular Angiography and Interventions. 2011 ACCF/AHA/SCAI Guideline for Percutaneous Coronary Intervention. A report of the American College of Cardiology Foundation/American Heart Association Task Force on Practice Guidelines and the Society for Cardiovascular Angiography and Interventions. J Am Coll Cardiol 58: e44-e122, 2011.

The Internal Medicine is an Open Access article distributed under the Creative Commons Attribution-NonCommercial-NoDerivatives 4.0 International License. To view the details of this license, please visit (https://creativecommons.org/licenses/ by-nc-nd/4.0/).

(C) 2017 The Japanese Society of Internal Medicine http://www.naika.or.jp/imonline/index.html 